\title{
Costa Rica sin Fronteras
}

Pensar en Movimiento:

Revista de Ciencias del Ejercicio y la Salud

ISSN 1659-4436

Vol. 11, No.1, pp. 1-2

\section{COSTA RICA SIN FRONTERAS \#7}

En esta sección se publican los resúmenes en español de artículos que han sido publicados por investigadoras/es de universidades costarricenses en otras revistas en el mundo, con su debida referencia al trabajo original, y con una breve explicación de dónde se realizó la investigación. Los artículos originales han sido publicados en otros idiomas; las revistas tienen consejo editorial y manejan un proceso de revisión por pares.

Los resúmenes corresponden a estudios que se conforman a los criterios generales de la revista, esto es, se trata de "... estudios experimentales o que hagan recomendaciones concretas para solucionar problemas o preguntas relevantes (...) trabajos originales o de meta-análisis." Solicitamos a las personas que han publicado este tipo de trabajos en otros idiomas que no los hagan saber, para incluir sus resúmenes aquí. 


\section{UN ESTUDIO DE CASOS Y CONTROLES DE LOS PATRONES DE ACTIVIDAD FÍSICA Y EL RIESGO DE INFARTO AL MIOCARDIO NO LETAL}

Gong, J., Campos, H., Fiecas, M. J. A., McGarvey, S. T., Goldberg, R., Richardson, C. \& Baylin, A. (2013). A case-control study of physical activity patterns and risk of non-fatal myocardial infarction. BMC Public Health, 13,122. doi:10.1186/1471-2458-13-122

Antecedentes: Los efectos interactivos de distintos tipos de actividad física en el riesgo de enfermedades cardiovasculares (ECV) no han sido plenamente considerados en estudios anteriores. Nuestro objetivo fue identificar aquellos patrones de actividad física que toman en cuenta distintas combinaciones de esta y examinar la relación entre esos patrones derivados de actividad física y el riesgo de infarto agudo al miocardio (IAM).

Metodología: Se examinó la relación entre los patrones de actividad física, identificados por análisis de componentes principales (ACP), y el riesgo de IAM en un estudio de casos y controles de infarto al miocardio en Costa Rica ( $N=4172)$, de 1994 a 2004. Se utilizaron los puntajes compuestos derivados del ACP y los METs totales para crear un modelo spline cúbico natural con el cual evaluar la asociación entre la actividad física y el riesgo de IAM.

Resultados: Se retuvieron cuatro patrones de actividad física del ACP caracterizados por: descanso o sueño, trabajo de agricultura, actividad liviana bajo techo y trabajo manual. Los patrones de actividad liviana bajo techo y de descanso o sueño mostraron una asociación lineal invertida ( $p$ para linearidad $=0.001$ ) y una asociación en forma de $U$ ( $p$ para nolinearidad $=0.03$ ) con el riesgo de IAM, respectivamente. Hubo una asociación invertida entre el gasto energético total asociado con la actividad y el riesgo de IAM, pero esta alcanzó una meseta en los niveles más altos de actividad física ( $p$ para no-linearidad $=0.01$ ).

Conclusiones: estos resultados sugieren que el patrón de actividad liviana bajo techo está asociado a un riesgo disminuido de IAM. EI ACP ofrece una nueva estrategia para investigar la relación entre la actividad física y el riesgo de ECV.

PALABRAS CLAVES: Patrones de actividad física, infarto al miocardio, Costa Rica

Este estudio es el fruto de la colaboración del Depto. de Salud Comunitaria de Brown University, el Depto. de Nutrición de la Escuela de Salud Pública de Harvard, el Depto. de Psiquiatría de la Universidad de California en San Diego, el Depto. de Ciencias Cuantitativas de la Salud de la Escuela de Medicina de la Universidad de Massachusetts, y el Depto. de Medicina Familiar así como el Depto. de Epidemiología de la Escuela de Salud Pública, ambos de la Universidad de Michigan. Los datos se recolectaron entre 1994 y 2004 en los hospitales del Valle Central en Costa Rica. La Dra. Hannia Campos concibió y diseñó el estudio junto con Ana Baylin. Colaboradora: Hannia Campos.

La revista BMC Public Health reporta un factor de impacto de 2.08 (año 2012).

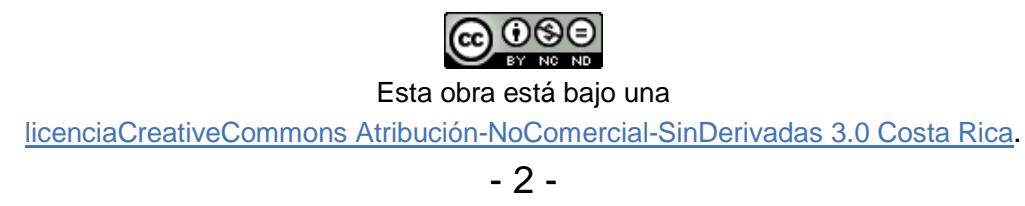

